\title{
e-Phaïstos
}

e-Phaïstos Revue d'histoire des techniques / Journal of the history of technology

VIII-2 $\mid 2020$

Aviation : émergence d'un complexe technique, 1900-1930

\section{De nouveaux horizons pour l'archéologie industrielle}

New Ways for Industrial Archaeology

\section{Marina Gasnier}

\section{(2) OpenEdition}

Journals

Édition électronique

URL : https://journals.openedition.org/ephaistos/7997

DOI : 10.4000/ephaistos.7997

ISSN : 2552-0741

Éditeur

IHMC - Institut d'histoire moderne et contemporaine (UMR 8066)

Référence électronique

Marina Gasnier, « De nouveaux horizons pour l'archéologie industrielle », e-Phaïstos [En ligne], VIII-2 I 2020, mis en ligne le 27 octobre 2020, consulté le 17 septembre 2021. URL : http://

journals.openedition.org/ephaistos/7997 ; DOI : https://doi.org/10.4000/ephaistos.7997

Ce document a été généré automatiquement le 17 septembre 2021.

Tous droits réservés 


\title{
De nouveaux horizons pour l'archéologie industrielle
}

\author{
New Ways for Industrial Archaeology
}

Marina Gasnier

\section{Définition et méthode}

1 L'archéologie industrielle naît en France à la fin des Trente Glorieuses, soit une vingtaine d'années après son apparition en Angleterre (Rix 1955 et 1967; Falconner 1980 et 2006). Étroitement lié au déclin industriel et à une prise de conscience de la disparition progressive de pans entiers d'activités humaines, ce nouveau champ disciplinaire a pour objectif l'étude et la valorisation des artéfacts issus de la civilisation industrielle - notamment sites de production et de transformation - à travers la collecte d'informations de nature hétérogène: archéologiques, écrites, iconographiques et orales. À l'instar des différentes branches de l'archéologie moderne et contemporaine, parmi lesquelles l'archéologie du bâti, la démarche ne recourt pas forcément à des campagnes de fouilles, même si certaines ont été menées assez tôt sur des sites tels ceux de la forge de Buffon (Côte-d'Or), de la forge d'Aube (Orne) ou encore des potiers de Saintonge (Charente-Maritime). En revanche, l'archéologie industrielle recourt systématiquement à un raisonnement théorique consistant à appliquer une méthode déductive destinée à reconstituer le fil d'une histoire ancrée dans le passé et à dénouer l'écheveau à partir de vestiges matériels, mais aussi d'observations de gestes techniques et de savoir-faire anciens. La dimension matérielle n'est donc pas exclusive, l'immatériel étant une composante essentielle du champ.

2 Par sa méthodologie rigoureuse, l'archéologie industrielle implique un travail de terrain et une pluridisciplinarité garantissant son originalité. Très vite, celle-ci a su dépasser l'étude des seules traces in situ pour s'ouvrir aux sciences sociales telles que l'anthropologie et l'ethnologie. Cependant, en France, l'archéologie industrielle doit sa structuration originelle aux historiens des techniques, de l'économie et de l'art. Parmi les précurseurs, il convient de rappeler le rôle déterminant de Maurice Daumas, à 
l'origine d'une vaste enquête sur les bâtiments à usage industriel aux $\mathrm{XVIII}^{\mathrm{e}}$ et $\mathrm{XIX}^{\mathrm{e}}$ siècles et auteur du premier ouvrage de référence sur le sujet (Daumas, 1980). Louis Bergeron, Serge Chassagne et Denis Woronoff lui emboîteront le pas et souligneront le bénéfice de recourir à ce nouveau champ pour renouveler les recherches dans le domaine de l'histoire des entreprises afin d'y porter un regard plus complet, non réductible à la seule exploitation de la documentation écrite. Dans ce contexte de réflexion intellectuelle poussée, caractéristique de l'émergence d'un nouveau champ, se posait la question de son appellation. Fallait-il préférer "patrimoine industriel » ou " archéologie industrielle ${ }^{1}$ "? L'histoire nous enseigne que la première formule fit consensus à l'issue des premières années d'étude ${ }^{2}$. En France, il fut convenu que l'archéologie industrielle concernait davantage la méthode d'investigation de cet objet naissant et captant de nouvelles attentions qu'était le patrimoine industriel. Davantage sémantique que scientifique, le débat était aussi motivé par la volonté de se démarquer de la terminologie d'origine britannique «industrial archaeology» majoritairement circonscrite aux vestiges matériels. Le choix français fut d'embrasser un spectre plus large en enrichissant ces mêmes artefacts de la dimension humaine et sociale que recouvre l'objet d'étude. Il semblait ainsi que la culture de l'industrie fut mieux représentée. Enfin, la préoccupation visait aussi la réception de ces termes par le grand public. Il fut convenu que le terme "archéologie » était plus réducteur, renvoyant au passé, tandis que celui de "patrimoine" semblait plus ouvert et plus à même de toucher la sensibilité des citoyens. La notion de transmission induite par le patrimoine n'était pas non plus étrangère à ce parti pris.

3 Près d'un demi-siècle plus tard, ce choix sémantique inscrit avec intelligence l'objet d'étude dans une continuité historique dont les opérations contemporaines de reconversion constituent une strate supplémentaire de ces sites en devenir ${ }^{3}$. Et même si le choix se fit à la faveur du patrimoine, il est incontestable que la démarche de l'archéologie industrielle, par essence pluridisciplinaire, semble la seule capable de garantir une approche exhaustive de l'objet, sans perdre de vue son épaisseur historique. Tenu entre la seconde moitié de la décennie 1970 et le début des années 1980, le débat aura ainsi permis d'engager une réflexion sur la patrimonialisation de l'industrie et de ses traces matérielles. Les travaux qui suivirent eurent des effets multiplicateurs en fédérant les institutions et les différents acteurs mobilisés en faveur de la sauvegarde de ce nouveau patrimoine. Parmi eux, diverses associations locales de défense œuvraient déjà vigoureusement de manière éparse au cœur des territoires lorsque le CILAC fut créé en $1979^{4}$ pour promouvoir l'objet au plan national. Parallèlement, l'État, en particulier le service de l'Inventaire général au ministère de la Culture, déploya en 1981 une politique nationale d'envergure consacrée, à partir de 1986, à un repérage exhaustif du patrimoine industriel, jusqu'à la décentralisation du service aux régions en 2007. À l'échelle internationale, le milieu associatif fut représenté par The International Committee for the Conservation of Industrial Heritage (TICCIH) fondé en 1973. Grâce à une mobilisation de la société civile combinée aux milieux institutionnel et académique, le patrimoine industriel a ainsi bénéficié des regards croisés d'experts en archéologie, en architecture, en histoire des techniques, en histoire économique et sociale, en ethnologie ou encore en anthropologie.

4 En termes méthodologiques, la pratique de terrain recouvre plusieurs vertus. Les artéfacts rencontrés peuvent prendre la forme d'objets archéologiques, d'outils, de produits manufacturés, d'infrastructures, de vestiges architecturaux ou bien d'édifices relativement bien conservés. Chacun de ces objets doit être interprété comme autant 
de marqueurs historiques de l'activité humaine qui s'est exercée à différentes périodes. Ils forment une succession de passerelles permettant d'entrer dans l'épaisseur de l'histoire et de mieux comprendre le sens du paysage industriel et technique dont nous héritons. Ce champ disciplinaire permet de saisir un ensemble productif et de réfléchir ensuite à la façon dont ses composantes in situ peuvent s'agencer non seulement à l'échelle du site, mais aussi de son environnement. Ce qu'il subsiste de ces ensembles est aussi révélateur de ce que le temps leur a ôté et, en ce sens, nourrit la valeur d'ancienneté du lieu chère à Aloïs Riegl, historien de l'art autrichien (Riegl 1903/1984).

Dans cette démarche, l'intérêt accordé aux fragments, à ce qui nous lie aux objets, $a$ priori insignifiants car relevant du quotidien, mais auxquels nous attribuons, dans le cas d'un processus de patrimonialisation, une importance similaire à celle que nous prêtons à l'art, implique un goût pour l'observation: savoir regarder les objets, le paysage, déceler les pleins comme les vides. La pratique du terrain est une voie particulièrement privilégiée pour se rapprocher de l'histoire des techniques. Par exemple, l'étude d'un paysage hydraulique en partie aménagé par l'homme, appréhendé par le prisme de relevés archéologiques, d'analyses architecturales ou d'équipements conservés, a tout à gagner en sollicitant, de manière complémentaire, la mémoire orale. C'est ainsi que je l'ai notamment expérimenté auprès d'anciens meuniers et minotiers dans le cadre d'une étude sur les moulins hydrauliques en Bretagne. La connaissance passe alors par la démonstration. Je me souviens encore de l'enthousiasme de ces derniers, fiers et heureux de partager leurs savoir-faire, leurs compétences techniques. Celles-ci sont d'ailleurs d'autant plus précieuses qu'elles compensent l'indigence des sources d'archives en la matière.

6 Le recours à la mémoire technicienne constitue une belle voie pour accorder au diapason théorie et pratique. Probablement plus qu'un document isolé, elle sait redonner à l'objet son «âme » et au-delà, son contexte technique, son rôle et sa place dans l'ensemble d'un procédé. L'enquête orale facilite la compréhension et donne du sens aux objets techniques. Sans elle, il faut savoir composer avec la ressource documentaire iconographique et écrite, ainsi qu'avec la conservation in situ des machines, des équipements de production, des moteurs énergétiques et/ou des objets fabriqués, dans une démarche de reconstitution historique et de va-et-vient entre le terrain et la littérature à disposition (brochures, encyclopédies, traités, etc.). Mais l'histoire des techniques s'illustre aussi dans la structure architecturale des ateliers témoignant de principes constructifs anciens. L'usine procède en effet de techniques de construction mettant en œuvre des matériaux vernaculaires (pierres de taille ou moellons en schiste, granite, etc.) ou bien issus de procédés industriels tels que la brique, le fer, la fonte, l'acier, le verre, le béton, ou encore de procédés ancestraux comme le torchis ou le pan de bois, là encore révélateurs d'un savoir-faire technique.

7 Du point de vue de leur organisation spatiale, les lieux de production, qu'ils relèvent du secteur artisanal ou du secteur industriel, recourent à une rationalité et à un agencement spécifique destinés à optimiser le process de fabrication et à faciliter les flux. La distribution concertée des ateliers les uns par rapport aux autres, leur implantation à proximité d'une source énergétique ou de matières premières, le fait de tirer avantage de la topographie des lieux - pour accroître le débit de l'eau sur les moteurs hydrauliques par exemple -, les rampes d'accès destinées à atteindre les gueulards des hauts fourneaux ou des fours à chaux, ou encore l'organisation des axes 
de circulation sont autant de données faisant appel à une intelligence technicienne. La cohérence du lieu émane de sa fonctionnalité productive.

Comparativement à un paysage hydraulique en milieu rural, l'enquête de terrain s'avère plus complexe au cœur des tissus urbains où l'architecture industrielle adopte un éventail typologique allant de l'îlot parcellaire parfaitement identifiable à la manufacture ou à l'atelier dont l'architecture mimétique se fond avec celle des façades mitoyennes et/ou environnantes. Toutefois, un œil avisé sait reconnaître un porche ou une cour intérieure trahissant l'activité passée des lieux, à l'abri des regards indiscrets. Alors que les historiens voient prioritairement dans l'enquête de terrain une ressource utile pour livrer des informations à travers l'observation des vestiges matériels, des traces laissées au sol, sur les murs, les ethnologues quant à eux ont une approche au plus près des hommes. L'une des conséquences de ces spécificités disciplinaires en est la fascination des uns pour les archives au détriment, parfois, des apports du terrain; puis la prédilection des autres pour l'architecture industrielle au détriment des équipements de production. Il apparaît effectivement manifeste que, en France, l'une des faiblesses du champ a été d'avoir souvent privilégié le patrimoine bâti au détriment du patrimoine technique. Plusieurs raisons expliquent cette orientation. En plus des acteurs déjà cités, l'émergence du champ doit aussi beaucoup aux photographies exposées et publiées à la fin des années 1970 sur le thème des «châteaux de l'industrie » à l'initiative de Maurice Culot, Lise Grenier et Hans Wieser-Benedetti, pour faire valoir une typologie de l'architecture industrielle de la région lilloise, celle des édifices éclectiques nés de l'industrie textile (Grenier, Wieser-Benedetti 1979). Si ces manifestations ont exercé un rôle incontestable dans la prise de conscience de la valeur patrimoniale de ces témoins matériels de l'industrie, cela s'est fait à travers une attention d'abord tournée vers l'architecture.

Une autre explication de ce travers semble provenir du profil souvent classique des agents du ministère de la Culture issu d'une formation en histoire de l'art. Cette hypothèse est d'autant plus probable qu'elle est confortée par l'intégration tardive, en 1994, des machines de production dans l'opération nationale de repérage du patrimoine industriel, soit huit ans après ses débuts. Bien entendu, la disparition massive des objets techniques et/ou machines de production, souvent vendus au prix de la ferraille, explique aussi cette sous-représentation de l'histoire des techniques pourtant essence même du patrimoine industriel. Malgré une évolution incontestable, notamment grâce à l'apport des technologies informatiques, ces dichotomies persistent encore... Pourtant, la combinaison des approches revendiquée par Louis Bergeron constitue l'une des exigences et l'originalité du champ qu'il faut savoir souligner, renforcer et revendiquer. Car malgré les faiblesses évoquées, il est indéniable que l'une des forces du patrimoine industriel a été de susciter un renouvellement de la conception classique de l'histoire économique, sociale et technique. Pour preuve, il suffit de mentionner les recherches consacrées, au fil de ces cinq dernières décennies, aux représentations et aux images de l'industrie ${ }^{5}$, celles dédiées à l'organisation, à la diffusion et à l'innovation technique ${ }^{6}$, ou encore celles qui ont affiné la monographie d'entreprise par une analyse globale combinant ces différentes entrées ${ }^{7}$. 


\section{Vers de nouveaux horizons...}

Après une dizaine d'années de travaux menés en archéologie industrielle, Denis Woronoff précisait, à juste titre, qu'il s'agissait, non pas d'une nouvelle discipline, mais d'une approche historique renouvelée de l'industrie (Woronoff 1989). Dans ce même texte, il en pointait les limites potentielles. Parmi elles, d'une part le risque de ne pas dépasser le stade de la monographie d'entreprise, et d'autre part la nécessité pour les chercheurs de « se comporter à l'égard de l'offre et de la demande sociales » (Woronoff 1989 : 447). Avec le recul qui est le nôtre aujourd'hui, cette double mise en garde se révéla fort bien pensée. Le risque monographique a été relativement évité grâce à des approches territoriales complémentaires dépassant l'échelle du site, ainsi qu'à des études thématiques. Les travaux universitaires engagés sur l'évolution des moteurs hydrauliques (L'Archéologie industrielle en France 1985), sur l'histoire du procédé indirect dans la fabrication du fer ainsi que sur sa diffusion (Woronoff 1984), puis d'autres menés par l'Inventaire général à l'échelle des territoires en témoignent (Gasnier 2018). Mais le risque persiste encore par une certaine difficulté à s'extraire du site pour l'inscrire dans une réflexion plus large, y compris dans le cadre d'une patrimonialisation. Au regard du capital de connaissances dont nous bénéficions aujourd'hui, force est de constater que ce fut effectivement le biais récurrent de l'approche historique et archéologique du patrimoine industriel. Mais ce constat ne réduit en rien l'apport incontestable de ces contributions dans la production du savoir, la compréhension de l'objet et l'extraordinaire richesse du champ dont témoigne la pluridisciplinarité des acteurs qui s'y sont intéressés. Chacun d'eux a donné une coloration spécifique au champ de l'archéologie industrielle à la française. La pluridisciplinarité qui était sienne à l'origine s'est renforcée au fil de sa lisibilité et des mutations sociétales. En effet, l'évolution culturelle, les modifications environnementales, les changements économiques ou encore les nouvelles politiques urbaines semblent avoir directement impacté les manières d'appréhender le patrimoine industriel.

11 Sous le coup de ces transformations importantes et d'une reconnaissance accrue, celuici a interpellé et suscité de nouveaux intérêts. La façon dont les sociétés gèrent la surenchère patrimoniale n'est pas non plus étrangère et conduit, de fait, à une observation de la part des uns et des autres. Depuis les années 2000, le regard des géographes a largement contribué à intégrer des angles d'approche complémentaires, parmi lesquels l'aménagement du territoire, le paysage, les représentations liées à la géographie sociale, culturelle et historique ${ }^{8}$. De l'approche de ces derniers, les historiens ont perçu tout l'intérêt scientifique de considérer les emboîtements d'échelle. En effet, les recherches consacrées au patrimoine industriel peuvent difficilement se réduire au périmètre du site. Indépendamment de l'angle d'attaque, qu'il s'agisse d'analyser les formes d'industrialisation ou de patrimonialisation, l'objet doit être étudié dans une réalité économique, sociale et culturelle, à l'échelle du quartier, de la ville, du territoire national ou international. Traité comme une donnée de la compréhension des sociétés, le patrimoine industriel sait aussi se renouveler sous la plume des sociologues (Rautenberg 2008 et 2013). Plus récemment, le recours aux outils numériques complète encore l'éventail. Contemporaine de l'émergence de la notion de "développement durable ", l'archéologie industrielle est, en outre, une voie d'analyse des dégradations environnementales superficielles, profondes, irréversibles, 
dont l'industrie est particulièrement responsable depuis deux siècles et que révèlent les empreintes écologiques.

\section{L'archéologie industrielle et l'environnement} chercheurs engagés dans l'étude du patrimoine industriel à être à l'écoute de «demande sociale » (Woronoff 1989 : 447) ? Il semblerait bien que les préoccupations écologiques actuelles constituent un ressort important en faveur de la préservation de ces objets patrimoniaux, pour peu que la recherche en archéologie industrielle s'engage dans cette voie afin d'en analyser le potentiel et, le cas échéant, de le faire valoir. Se concentrer sur la reconversion des anciens lieux de l'industrie sous l'angle interdisciplinaire est une manière de renouveler les termes du questionnement, comme de recourir à de nouvelles méthodes de travail sans perdre de vue la cohérence qui doit s'exercer avec les acquis, les textes doctrinaux et les besoins contemporains.

$\mathrm{Au}$ regard du contexte actuel, le patrimoine industriel n'est plus seulement le témoin historique d'une époque, d'un style architectural, d'un processus technique. Il recouvre désormais un rôle actif dans la production du territoire, celui d'un levier entre le passé, le présent et l'avenir. L'enjeu est de nourrir la connaissance, mais aussi d'encourager l'appropriation et la gestion de ce patrimoine dans le respect de ses valeurs. Les perspectives ne sont plus seulement celles des secteurs culturels et touristiques. Elles touchent les enjeux économiques et environnementaux (Gasnier 2018). La mise en correspondance des politiques de la ville, des stratégies d'économie d'énergie et de développement territorial avec la démarche de l'archéologie industrielle est une voie tout entière à dessiner. L'ouverture à d'autres sphères, ainsi que le changement d'échelle à laquelle est traité le patrimoine industriel ont une incidence directe sur sa perception sociale et, le corollaire, la place qui lui est alors accordée. L'une des manières de participer à l'exploration de ces pistes est l'interdisciplinarité entre les sciences historiques et les sciences de l'ingénierie. Car si la pluridisciplinarité qui était sienne dès l'origine, concentre, autour d'un même objet, une variété d'approches, l'interdisciplinarité va plus loin dans la mesure où elle invite à un transfert des méthodes d'une discipline à l'autre. Autrement dit, l'interaction est plus étroite et dynamique.

L'intérêt de cet enrichissement réciproque est double. D'abord, il s'agit de développer la recherche fondamentale et d'accroître la connaissance par des voies jusqu'alors inexplorées. Ensuite, l'interdisciplinarité est une piste inédite invitant au renouvellement épistémologique du champ et à la mise à jour de nouveaux axes de travail. Mais aborder cet objet sous le prisme de la question environnementale revient aussi à souligner leur relation particulière. D'une part, l'industrialisation massive comme fait et cause de l'épuisement des ressources naturelles et de la pollution constatée à l'échelle internationale ; d'autre part, le réemploi de certains de ses artéfacts comme acte contribuant, à leur échelleq à freiner le processus de dégradation de l'environnement. C'est encore une manière de revisiter les perspectives des premiers travaux historiques soulignant la dialectique étroite entre les ressources naturelles et les activités de l'homme. Qu'elles soient minérales, forestières ou hydrauliques, ces ressources ont joué, jusqu'au Second empire, un rôle déterminant dans l'implantation des établissements industriels. Puis, le développement des 
infrastructures routières, ferroviaires et navigables réduisît le coût du transport des matières premières et $d u$ combustible, en même temps que l'accroissement du machinisme libéra les usines de leur dépendance énergétique hydraulique. Si l'industrie a parfois aidé à réguler certaines de ces ressources dont le domaine forestier, elle les a surtout extraites, exploitées, transformées, consumées, laissant dans le paysage des stigmates dont certains ont acquis une valeur symbolique. À cet égard, l'inscription du bassin minier du Nord-Pas-de-Calais au titre de patrimoine mondial de l'UNESCO ${ }^{10}$ témoigne d'une évolution de la perception de ce paysage industriel (Chaplain-Manigand, Guido 2014; Crouzet 1997) au sein duquel les terrils ont changé d'image. Le statut de «monument » s'est substitué au monticule de déchets miniers.

Cette mutation sociale et culturelle contemporaine n'est pas sans faire écho à la valeur d'ancienneté (Riegl 1903/1984) pour laquelle le poids de l'affect, proportionnel à l'égrenage du temps, modifie les regards sur l'objet, le monument, l'artéfact, la trace. Ce contexte invite ainsi à penser notre relation à l'environnement autrement que sous l'angle de la culpabilité collective, mais davantage dans une perspective de réconciliation et de co-construction que suggère le concept de durabilité, des points de vue paysager et architectural. Le nouveau paradigme écologique rend de plus en plus pressant le développement de telles recherches, l'élaboration d'outils et l'invention de nouvelles méthodes visant à interroger la capacité de cet héritage bâti à servir les exigences environnementales, dans le respect de ses valeurs patrimoniales. L'originalité tient dans l'interdisciplinarité croisant les sciences humaines et sociales (histoire ; archéologie industrielle ; architecture ; urbanisme ; histoire de l'art) et les sciences pour l'ingénieur (thermique; mécanique). Si l'homme a toujours entretenu un lien étroit avec la nature dans le cadre de ses activités de production (rivières et mers comme ressource énergétique; bois des forêts comme combustible; extraction des ressources minérales, etc.) dans des conditions où l'équilibre des écosystèmes n'a pas toujours été respecté, le patrimoine industriel en constitue aujourd'hui une des traces tangibles, mais aussi un des ressorts qu'il est nécessaire d'évaluer et de réinvestir à la faveur de la cause environnementale. Dans le contexte de la transition énergétique et de ses conséquences sur le bâti existant, l'une des pistes est de mener une réflexion sur les propriétés hygrothermiques des édifices industriels anciens dont la reconversion est un des défis de nombreuses collectivités. Il devient en effet urgent d'accroître la connaissance des matériaux anciens pour optimiser la qualité des diagnostics et contribuer ainsi à une meilleure sauvegarde des bâtiments. La fiabilité des diagnostics grâce au croisement des compétences en ingénierie thermique et mécanique, ainsi qu'en sciences historiques et patrimoniales, permettrait d'éviter de recourir à des solutions inadaptées voire irréversibles en matière de préservation patrimoniale.

Des recherches sont actuellement en cours à l'Institut FEMTO-ST (UMR 6174), pilotées par deux équipes aux compétences complémentaires en thermique et en sciences historiques et du patrimoine ; l'un des axes d'étude concerne le développement d'une méthodologie non destructive d'évaluation des performances hygrothermiques de matériaux anciens, en l'occurrence la brique (El Mankibi et al. 2019). Parallèlement, il est bon de mentionner la récente évolution historiographique inscrite dans le prolongement des travaux d'André Guillerme ${ }^{11}$ et caractérisée par le rapprochement entre l'histoire des techniques et l'histoire environnementale. Sans qu'il soit véritablement le cœur du propos, le patrimoine industriel apparait de manière différenciée dans les recherches de cette nouvelle génération de chercheurs ${ }^{12}$ spécialisés dans le domaine de l'histoire des nuisances, des pollutions et de la 
composition des tissus industriels. Si la question de la pollution industrielle est délicate compte tenu de ses conséquences sanitaires et environnementales, elle peut aussi être abordée en des termes renouvelés liés au développement technologique. Le contexte de la reconversion patrimoniale à d'autres usages génère des expérimentations sur site où la bio-technologie conduit à envisager le rapport nature-industrie et les traces anthropiques sous un angle tenant à l'écart les discours culpabilisants ${ }^{13}$. À titre d'exemple, des expériences de phyto-remédiation, de dépollution par les plantes sont menées sur d'anciens sites industriels textiles et chimiques ${ }^{14}$. Parallèlement, il n'est pas rare de constater qu'une biodiversité très spécifique peut aussi se développer sur des terrils; de même, d'anciens sites chaufourniers au sous-sol calcaire sont particulièrement propices au développement d'orchidées rares (Fours à chaux de Lormandière, Ille-et-Vilaine). Loin de nier la question ardue de la pollution des sols, ce nouveau rapport de l'industrie à la nature s'illustre, par exemple, par le classement d'anciens carreaux de fosse en Espaces Naturels Sensibles (bassin minier du Nord-Pasde-Calais).

17 Il convient aussi de considérer l'angle législatif, le nouvel usage conditionnant le degré de dépollution à envisager. En milieu urbain, dans un contexte de réappropriation positive de l'espace, les anciens sites industriels placés en bordure de rivière, fleuve ou front de mer, sont traités comme de futurs espaces d'aménité revendiqués au titre d'un urbanisme de qualité lors des opérations d'aménagement et de reconversion patrimoniale (Ile de Nantes; Euroméditerranée à Marseille; Saint-Dizier; Lyon; Bordeaux ou encore Rouen). Ces stratégies contemporaines montrent que la question environnementale peut être appréhendée sous le double prisme de la valorisation du patrimoine industriel, au titre de support mémoriel, et de la reconquête conjointe des espaces par la nature. Bien évidemment, si la patrimonialisation ne dédouane pas le site industriel de son histoire passée, ni de son impact sur l'environnement, en revanche, elle peut révéler un sens des responsabilités à l'égard du territoire par le traitement des lieux lors de leur reconversion. Celle-ci renvoie à la recomposition des lieux, à la reconstruction des villes sur elles-mêmes par la voie patrimoniale. Les anciens sites industriels sont le support d'un empilement de couches archéologiques comme autant de traces de leur évolution historique. Chacune d'elle a valeur symbolique dans la mesure où elles sont toutes associées à un contexte spécifique que l'homme a généré, autant qu'il s'y est adapté. Des édifices tels que l'Alhambra de Grenade et la Mezquita de Cordoue sont les paradigmes d'une architecture qui se transforme dans le temps comme a parfaitement su le distinguer l'urbaniste italien Aldo Rossi. Ils incarnent une architecture constitutive de la ville, à l'image de certains sites industriels qui ont généré des quartiers entiers, sinon des villages ou des villes. Il semble ainsi nécessaire de considérer avec attention les associations possibles et évolutives entre les objets et les situations. En ce sens, Aldo Rossi s'exprime en ces termes: "L'émergence des relations entre les choses, plus que les choses elles-mêmes, instaure toujours de nouvelles significations » (Rossi 1981/1988:36).

Porter un intérêt à la reconversion, au réemploi du bâti existant et aux processus de patrimonialisation avec l'œil de l'archéologue, implique de s'intéresser de près au concept de continuité dans laquelle s'inscrivent ces choses, et au concept de renouvellement que ces mêmes choses suscitent à la lueur des contextes distincts de celui qui les a faits naître. En ce sens, le contexte de la transition énergétique pourrait bien être une opportunité pour insuffler un nouveau souffle à l'étude du patrimoine industriel. Évolution des « valeurs » pour Aloïs Riegl, «nouvelles significations » pour 
Aldo Rossi... Il est question de perméabilité et de réceptivité entre les objets, l'espace et le temps.

\section{L'archéologie industrielle et les humanités numériques}

19 Du point de vue épistémologique, il semble tout autant légitime de s'interroger sur l'apport potentiel des humanités numériques dans un contexte d'analyse et de valorisation du patrimoine industriel et technique. Par humanités numériques, il est ici question d'articuler les problématiques et les méthodes issues des sciences humaines et sociales, de la statistique et de l'informatique (CAO, base de données, méthodes quantitatives, etc.). Il s'agit d'interroger plus largement le tournant numérique pris par la société et ses conséquences sur les conditions de production et de diffusion des savoirs. Cet angle d'approche permet, grâce au croisement des outils, d'enrichir l'objet d'étude, de le questionner autrement, voire d'en faciliter l'analyse et la diffusion. Dès lors, recourir aux outils informatiques recouvre plusieurs enjeux parmi lesquels la promotion de la culture scientifique, technique et industrielle, mais encore le développement de l'interdisciplinarité entre les sciences pour l'ingénieur et les sciences humaines et sociales.

En ce sens, le champ récent de "l'archéologie industrielle avancée " (Laroche 2007) consiste à partir du présent pour remonter le passé en croisant les sources d'archives et les observations de terrain, mais aussi à enrichir ces données historiques par l'utilisation des outils numériques, notamment dans une démarche de rétroconception. Adaptation de la notion de reverse-engineering utilisée en industrie par les ingénieurs, celle-ci vise à reconstituer en 3D l'objet technique, la machine ancienne à partir de logiciels de CAO afin d'en restituer le fonctionnement, la cinématique. En matière de patrimoine technique, les fermetures d'usines se soldent souvent par la disparition de leurs machines, assez fréquemment ferraillées ou vendues à des pays émergents. Lorsqu'elles sont préservées, il n'est pas rare de les voir sorties de leur contexte historique pour être érigées symboliquement à l'entrée d'une ville. Aussi, sans envisager une quelconque substitution, la question délicate de l'absence de conservation in situ du patrimoine technique s'est enrichie de nouvelles perspectives grâce aux technologies numériques ${ }^{15}$. Celles-ci constituent une manière de penser autrement les temporalités de l'industrie par la modélisation 3D des objets techniques anciens, voire la réalité augmentée. En plus de constituer un support de médiation culturelle et de valorisation patrimoniale, ce type de produit numérique apporte des éléments de compréhension complémentaires aux systèmes techniques traditionnellement étudiés à travers les archives écrites et iconographiques.

21 En croisant ainsi les compétences des sciences humaines et sociales à celles des sciences pour l'ingénieur, la méthode discrimine la complexité structurelle de l'objet technique et améliore la connaissance. De nouvelles clés de lecture sont proposées par ces voies tout entières à explorer et à développer. Bien entendu, si ces outils ne peuvent se substituer à la conservation des objets, en revanche ils sont une réponse tangible aux questions que soulevait déjà Aloïs Riegl dans son essai sur les monuments historiques, lorsqu'il imaginait une reconstitution en images, en pensée ou en paroles des œuvres disparues (Riegl 1903: 51). Mais au-delà du seul maquettage numérique et de la restitution 3D d'un objet et de sa cinématique, la force des humanités numériques serait d'aborder l'histoire des techniques dans la longue durée afin d'en saisir 
l'évolution, la génétique, les éventuels épisodes de rupture et/ou de mutation. En ce sens, une analyse diachronique des gestes techniques, des outils, puis leur adaptation par la mécanisation semble particulièrement riche et pertinente. Comme l'indiquait René Rémond, la perception de la continuité est une manière de prendre conscience de l'Histoire, notamment par la reconnaissance d'un héritage. J'interprète cet héritage comme pouvant être de différente nature, en l'occurrence le legs de l'industrie: matériel et immatériel.

L'idée est donc de recourir aux outils informatiques pour rendre compte du transfert des connaissances techniques. Comme Yohann Beckmann l'a fait deux siècles auparavant dans le cadre de la parution, en 1806, de son ouvrage intitulé « Entwurf der algemeinen " (Projet de technologie générale) (Carnino, Hilaire-Pérez et Hooc 2017), il semble tout à fait prometteur de s'inscrire dans son sillage avec les outils de notre temps afin de formaliser la transformation et la production technique, non pas par la taxinomie, mais par la modélisation 3D. En quoi l'usage du maquettage numérique modifie-t-il notre rapport aux objets techniques ? Comment l'outil numérique peut-il aider à se réapproprier les gestes techniques et les mécanismes anciens, ainsi qu'à en percevoir l'héritage, la filiation, les transferts dans le temps long ? Le questionnement est double et porte non seulement sur l'apport des technologies numériques dans la connaissance et la gestion des données, mais aussi sur la transformation des usages qu'elles suscitent. En quoi ces outils peuvent-ils contribuer autrement que par une démarche historique traditionnelle à l'analyse diachronique des gestes, des savoirfaire, des pratiques, de la mémoire procédurale?

À l'articulation de l'enseignement et de la recherche, et au carrefour de nombreuses disciplines ou champs disciplinaires (histoire, archéologie industrielle, informatique appliquée, génie mécanique, cliométrie, intelligence artificielle, réalité virtuelle, sociologie et géomatique), le projet « Techn'hom Time Machine » développé à l'Université de technologie de Belfort-Montbéliard envisage une reconstitution multidimensionnelle d'un ancien quartier industriel implanté à Belfort à la fin du XIX siècle. Au fur et à mesure de la restitution en 3D, ce processus d'écriture de l'histoire a pour objectif de répondre à ce type de questionnements tout en stimulant les pratiques en ingénierie, patrimoine et numérique. Dans le cadre de ce projet, le milieu gestuel doit être considéré dans sa totalité afin d'en extraire les rationalités, les ruptures, les continuums. Il est question de «technologie opératoire» comme l'entendait J. Beckmann, à savoir une analyse des pratiques, des opérations. À travers l'étude fine de ces dernières, l'approche est aussi systémique et considère tant les hommes, les outils, les machines, les ateliers, les flux, le site, que le quartier ou le territoire.

Les humanités numériques sont ainsi une orientation prometteuse pour générer de nouvelles formes de relations avec l'héritage patrimonial en tant qu'objet d'étude, mais aussi pour déployer un éventail d'outils favorisant la production de la connaissance, sa valorisation et sa diffusion. Un rapide regard rétrospectif permet de rappeler que les premiers travaux de rétroconception d'objets techniques anciens ont été initiés à l'Université de technologie de Belfort-Montbéliard au début des années 2000, en particulier par Michel Cotte, professeur honoraire d'histoire des techniques et Florent Laroche ${ }^{16}$. Depuis, ces recherches bénéficient d'actions complémentaires, parmi lesquels il faut notamment compter la collaboration de Jean-Louis Kerouanton et Sylvain Laubé au Centre François Viète (Université de Nantes/Brest); les travaux de l'équipe « Usines 3D » de l'université d'Evry avec Alain-P. Michel et Robert Vergnieux 
(en particulier Virtual Retrospect, 2009) ; les activités de l'équipe d'Archéovision (UMS 3657) à l'Université de Bordeaux ; ou encore celles qui se poursuivent à l'Université de technologie de Belfort-Montbéliard ${ }^{17}$. L'apport du numérique dans l'étude et la valorisation du patrimoine industriel et technique peut être extrêmement intéressant dès lors que les outils utilisés et les démarches adoptées ne se réduisent pas à une dimension cosmétique. La dynamique de recherche engagée dans ce domaine bénéficie de quelques foyers actifs dont témoignent le projet ANR ReSeed ${ }^{18}$ et Lab In Virtuo ${ }^{19}$, en cours. D'autres perspectives sont à inventer pour enrichir, élargir et renouveler les approches de l'archéologie industrielle. Une réflexion est notamment en cours sur le concept de patrimoine industriel augmenté dans le cadre du projet Techn'hom Time Machine ${ }^{20}$.

\section{En guise de conclusion, un regard personnel}

Face à cet objet d'étude qu'est le patrimoine industriel, la démarche que je tente d'adopter correspond à la perception que je me fais du métier d'historien, de sa vocation sociale et de sa responsabilité morale à l'égard de certains enjeux de son temps. L'érudition, la connaissance, l'accumulation de savoirs ne peuvent être exclusives des préoccupations sociétales. L'équilibre est nécessaire. À ce titre, j'appartiens à cette frange de la communauté scientifique engagée dans la rechercheaction. Cette démarche me conduit à participer à des expertises ou à assurer des interventions auprès d'acteurs relevant des collectivités, chambres de commerce et d'industrie, agences de développement et d'urbanisme, ou encore sociétés d'économie mixte. Dans cette perspective, le rôle de l'historien est aussi celui de l'animation et de la valorisation. Alors qu'elle prend de plus en plus d'ampleur à l'échelle internationale, la question environnementale amène à reconsidérer la responsabilité de chacune et de chacun d'entre nous. Celle-ci ne peut incomber au seul politique qui souhaite œuvrer en ce sens. Cette responsabilité est collective et doit opérer à la plus petite échelle.

En septembre 2013, la conférence inaugurale des Journées de l'Architecture organisées par la Maison Européenne de l'Architecture - dispensée par Wang Shu, architecte chinois (Prix Pritzker 2012), conforta, d'une part mon regard sur l'alliance entre le passé et la modernité ; d'autre part mon positionnement relatif à la valeur de l'objet patrimonial, en l'occurrence industriel, face à l'uniformisation des paysages. Par l'intérêt qu'il porte à la métamorphose de l'architecture traditionnelle et contemporaine, de la ville et du paysage, Wang Shu envisage le changement en incluant les valeurs héritées du passé, tel qu'il est souhaitable pour la reconversion du patrimoine industriel. À travers plusieurs expérimentations menées avec ses étudiants, puisqu'il enseigne aussi à l'Université d'Art de Chine à Hangzhou, l'architecte réfléchit notamment à l'utilisation conjointe du bambou et du béton. Il envisage l'acte architectural à travers le recyclage, non seulement des matériaux, mais aussi des techniques, des savoir-faire traditionnels ${ }^{21}$. Très attentive à la question de l'utilisation des ressources et à celle de la standardisation des paysages bâtis, je pense que le maintien de la diversité architecturale est essentiel. La qualité paysagère est en jeu, comme l'est l'identité territoriale. En plus d'être vertueux, l'intérêt de la reconversion du patrimoine est double : architectural et paysager. La préservation des valeurs et du bâti hérités du passé est une contribution à cette variété, en plus d'être un acte de développement soutenable. Il est tout à fait clair que la reconversion du patrimoine 
industriel répond à l'ensemble de ces préoccupations. Pourtant, face au dérèglement climatique, à la pression démographique, à la densification et aux multiples restructurations urbaines, nous assistons encore trop souvent à des politiques de la table rase privilégiant la destruction d'édifices patrimoniaux pour construire des architectures insipides, génériques, uniformes d'un territoire à l'autre.

C'est pourquoi, au titre de la valorisation de la permanence et de la prise en compte de l'Histoire comme un continuum, ma responsabilité à l'égard de l'avenir est aussi essentielle que mon désir de voir les héritages du passé préservés dans une intelligence collective. Au cœur de la reconversion patrimoniale se jouent l'affirmation des identités territoriales et la reconnaissance d'une alliance intime, dans la durée, entre l'héritage du passé et la modernité, entre la tradition et le renouveau, par les matériaux, les techniques. Continuité et renouvellement entretiennent un dialogue étroit dans le déroulement du palimpseste de l'Histoire. Et face aux enjeux écologiques et numériques, le patrimoine industriel constitue un objet d'étude que les jeunes générations de chercheurs doivent savoir renouveler afin de maintenir ce lien entre le passé et les préoccupations sociétales de demain.

\section{BIBLIOGRAPHIE}

ANDRÉ Louis, Machines à papier. Innovation et transformations de l'industrie papetière en France, 1798-1860, Paris, Éditions de l'EHESS, 1996

BALUT Pierre-Yves, «L'archéologie buissonnière : 2. L'archéologie industrielle ", Ramage, $\mathrm{n}^{\circ} 3$, 1984-1985, p.243-258, http://www.tetralogiques.fr/lirl/revues/ramage.html

BELOT Robert et LAMARD Pierre, Peugeot à Sochaux. Des hommes, une usine, un territoire, Paris, Lavauzelle, 2007

BELOT Robert, LAMARD Pierre (dir.), Alstom à Belfort. 130 ans d'aventure industrielle, BoulogneBillancourt, ETAI, 2009

BELOT Robert, LAMARD Pierre, Images de l'industrie, XIX ${ }^{e}$-XX $X^{e}$ siècles, Antony, ETAI, 2011

CARDINAL Catherine, HILAIRE-PÉREZ Liliane, SPICQ Delphine et THÉBAUD-SORGER Marie (dir.), «L'Europe technicienne XV ${ }^{\mathrm{e}}$-XVIII" siècle », Artefact. Techniques, histoire et sciences humaines, $\mathrm{n}^{\circ} 4$, 2016, https://doi.org/10.4000/artefact.275

CARNINO Guillaume, HILAIRE-PEREZ Liliane, HOOC Jochen (dir.), Johann Beckmann, Entwurf der algemeinen Technologie / Projet de technologie générale (1806), Rennes, PUR, 2017

CHAPLAIN-MANIGAND Catherine et GUIDO Anthony (coord.), Les paysages de l'industrie, Compte rendu des journées d'études à l'INSET de Montpellier, Mission de l'Inventaire général du patrimoine culturel, 2014

CHASSAGNE Serge, «L'élargissement d'un concept : de l'archéologie (industrielle) au patrimoine (industriel) », Le Mouvement Social, 2-199, 2002, p.7-9, https://doi.org/10.3917/lms.199.0007 
COTTE Michel, « Les techniques numériques et l'histoire des techniques. Le cas des maquettes virtuelles animées », Documents pour l'histoire des techniques, n¹8, 2009, p. 7-21, http:// journals.openedition.org/dht/97

COTTE Michel, LAROCHE Florent, Le virtuel pour capitaliser notre patrimoine technique et industriel, in ICOMOS 17th General Assembly, 2011-12-02, Paris, http://openarchive.icomos.org/id/ eprint/1325

COTTE Michel, «Les outils numériques au service de l'histoire des techniques », e-Phaïstos, revue d'histoire des techniques, I-2, 2012, p.12-27, https://doi.org/10.4000/ephaistos.392

COTTE Michel, « Le patrimoine technique de l'époque moderne, aperçu d'un monde en transition ", Artefact. Techniques, histoire et sciences humaines, ${ }^{\circ} 4$, 2016, p.371-383, http:// journals.openedition.org/artefact/528

CROUZET François, « Naissance du paysage industriel », Histoire, économie et société, vol.16, n³, 1997, p.419-438, https://doi.org/10.3406/hes.1997.1955

DAUMAS Jean-Claude, Les territoires de la laine. Histoire de l'industrie lainière en France au XIX ${ }^{e}$ siècle, Villeneuve d'Ascq, PUS, 2004

DAUMAS Jean-Claude (dir.), La mémoire de l'industrie. De l'usine au patrimoine, Besançon, PUFC, 2006

DAUMAS Maurice, L'archéologie industrielle en France, Paris, Laffont, 1980

DAUMAS Maurice, «L'archéologie industrielle : ses méthodes, ses succès, ses limites ", Ramage, 1, 1982, p.37-47

DI MEO Guy, « Patrimoine et territoire, une parenté conceptuelle », Espaces et Sociétés, vol.78, n4, 1994, p.15-34, https://www.cairn.info/revue-espaces-et-societes-1994-4-page-15.htm

Documents pour l'histoire des techniques, CNAM/CDHT et CDHTE, DOI : https://doi.org/10.3406/rhs. 1963.4527, et pour la nouvelle série : https://journals.openedition.org/dht/

EDELBLUTTE Simon, « Paysages et territoires du patrimoine industriel au Royaume-Uni », Revue Géographique de l'Est, vol.48, n¹-2, 2008, http://journals.openedition.org/rge/1165

EDELBLUTTE Simon, Paysages et territoires de l'industrie en Europe : héritages et renouveaux, Paris, Ellipses, 2010

El MANKIBI Fatima, AIT OUMEZIANE Yacine, LEPILLER Valérie, GASNIER Marina, DESEVAUX Philippe, « Procédure expérimentale appliquée à l'identification des propriétés hygrothermiques des matériaux de construction par méthode non destructive ", Actes du Congrès annuel de la Société Française de Thermique, Nantes, 2019, p.817-824

FALCONER Keith, Guide to England's industrial heritage, New York, Holmes \& Meier, 1980

FALCONER Keith, " The industrial heritage in Britain. The first fifty years ", La revue pour l'histoire $d u$ CNRS [En ligne], 14, 2006, https://doi.org/10.4000/histoire-cnrs.1778

GARAT Isabelle, GRAVARI-BARBAS Maria et VESCHAMBRE Vincent, «Émergence et affirmation du patrimoine dans la géographie française : la position de la géographie sociale ", in Jean-Marc FOURNIER (dir.), Faire de la géographie sociale aujourd'hui, Les Documents de la Maison de Recherche en Sciences Humaines de Caen, 14, 2001, p.31-40

GARÇON Anne-Françoise, Mine et métal. 1780-1880 les non-ferreux et l'industrialisation, Rennes, PUR, 1998

GARÇON Anne-Françoise et HILAIRE-PEREZ Liliane (dir.), Les chemins de la nouveauté : innover, inventer au regard de l'histoire, Paris, CTHS, 2004 
GASNIER Marina, Le patrimoine industriel au prisme de nouveaux défis. Usages économiques et enjeux environnementaux, Besançon, PUFC, 2018

GASNIER Marina, « Réflexion épistémologique sur le patrimoine industriel. De la pluridisciplinarité à l'interdisciplinarité ", Revue d'Histoire des Sciences, 72-2, juillet-décembre 2019, p.309-348, https://doi.org/10.3917/rhs.722.0309

GAY Georges, «L'impossible palimpseste industriel », L'Archéologie industrielle en France, n61, 2012, p.11-19

GIEC, « Changements climatiques 2014 », $5^{e}$ rapport d'évaluation du Groupe d'experts intergouvernemental sur l'évolution du climat, 2014, https://www.transition-europe.eu/fr/ publication/5eme-rapport-devaluation-du-giec-sur-les-changements-climatiques-et-leursevolutions

GRAVARI-BARBAS Maria, GUICHARD-ANGUIS Sylvie (dir.), Regards croisés sur le patrimoine dans le monde à l'aube du XXI ${ }^{e}$ siècle, Paris, Presses de l'Université Paris-Sorbonne, 2003

GRENIER Lise, WIESER-BENEDETTI Hans, Recherches sur l'architecture de la région lilloise de 1830 à 1930 : Les châteaux de l'industrie, Paris-Bruxelles, Archives d'architecture moderne et ministère de l'Environnement et du Cadre de Vie, 1979

HERVY Benjamin, Contribution à la mise en place d'un PLM muséologique dédié à la conservation et la valorisation du patrimoine : modélisation et intégration de données hétérogènes sur un cycle de vie produit complexe, thèse en sciences de l'ingénieur [physics], École Centrale de Nantes, 2014

HILAIRE-PEREZ Liliane, L'invention technique au Siècle des Lumières, Paris, Albin Michel, 2000

L'Archéologie industrielle en France, $\mathrm{n}^{\circ}$ 49, 2007, https://www.cilac.com/aif-numero-49-fevrier-2007

L'Archéologie industrielle en France, $\mathrm{n}^{\circ}$ 45, Actes du colloque CILAC, Le Creusot, 23/26 septembre 2004, 2004, https://www.cilac.com/aif-numero-45-decembre-2004

LAROCHE Florent, Contribution à la sauvegarde des objets techniques anciens par l'archéologie industrielle avancée. Proposition d'un modèle d'information de référence muséologique et d'une méthode interdisciplinaire pour la capitalisation des connaissances du patrimoine technique et industriel, thèse de doctorat en génie mécanique, École Centrale de Nantes, I, 2007

LAROCHE Florent, COTTE Michel, KEROUANTON Jean-Louis, BERNARD Alain, « L'image virtuelle comme source de connaissance pour le patrimoine technique et industriel : Comment allier Histoire et Ingénierie? », Congrès national des sociétés historiques et scientifiques, Arles, 2007, CTHS, 2009, p.1-12, https://hal.archives-ouvertes.fr/hal-00422187

LOHR Evelyne, MICHEL Geneviève, PIERROT Nicolas (dir.), Les grands moulins de Pantin. L'usine et la ville, Lyon, Lieux-Dits, 2009

MICHEL Alain P., « La reconstitution virtuelle d'un atelier de Renault-Billancourt : sources, méthodologie et perspectives ", Documents pour l'histoire des techniques, 18, 2009, p.23-36, http:// journals.openedition.org/dht/198

OLIVIER Jean-Marc, Des clous, des horloges et des lunettes. Les campagnards moréziens en industrie (1780-1914), Paris, CTHS, 2004

PIERROT Nicolas, Les images de l'industrie en France : peintures, dessins, estampes, 1760-1870, thèse de doctorat en histoire des techniques, Centre d'Histoire des Techniques/IHMC, Université Paris 1 Panthéon-Sorbonne, 2010, 2 vol. 
PONS-BRANCHU Edwidge, GUILLERME André, « Urbanization impact on sulfur content of groundwater revealed by the study of urban speleothem-like deposits: Case study in Paris, France ", Science of the Total Environment, 579, feb. 2017, p.124-132

RAUTENBERG Michel, « Patrimoines culturel et naturel : analyse des patrimonialisations », Culture et Musées, hors-série (La muséologie, 20 ans de recherches : Actes Sud, 2013), p.115-138, https://doi.org/10.4000/culturemusees.734

RAUTENBERG Michel, Stratégies identitaires de conservation et de valorisation du patrimoine, Paris, éditions L'Harmattan, 2008

RIEGL Aloïs, Le culte moderne des monuments. Son essence et sa genèse, Paris, Seuil, (1903) 1984

RIX Michael, « Industrial Archaeology », The Amateur Historian, 2(8), 1955, p.225-229

RIX Michael, Industrial Archaeology, London, Historical Association, 1967

ROSSI Aldo, Autobiographie scientifique, traduit de l'italien par Catherine Peyre, Marseille, Éditions Parenthèses, (1981) 1988

SMITH Paul (dir.), « La reconversion des sites et bâtiments industriels », In Situ [en ligne], 26, 2015, URL : http://insitu.revues.org/11744

VESCHAMBRE Vincent, « Patrimoine : un objet révélateur des évolutions de la géographie et de sa place dans les sciences sociales », Annales de géographie, vol.656, n4 , 2007, p.361-381, https:// doi.org/10.3917/ag.656.0361

VESCHAMBRE Vincent, Traces et mémoires urbaines. Enjeux sociaux de la patrimonialisation et de la démolition, Rennes, PUR, 2008

WORONOFF Denis, L'industrie sidérurgique en France pendant la Révolution et l'Empire, Paris, éditions de l'EHESS, 1984

WORONOFF Denis, «L'archéologie industrielle en France : un nouveau chantier », Histoire, économie et société, 8-3, 1989, p.447-458, https://doi.org/10.3406/hes.1989.1539

WORONOFF Denis, La France industrielle : gens des ateliers et des usines, 1890-1950, Paris, éditions du Chêne, 2003

WORONOFF Denis, Histoire de l'emballage en France du XVIII siècle à nos jours, Presses universitaires de Valenciennes, 2015

\section{NOTES}

1. Celle-ci fit la préférence des précurseurs anglais qui se sont appropriés le champ dès le milieu des années 1955 : Michael Rix, de l'université de Birmingham et Kenneth Hudson, Senior Lecturer in Adult Studies à l'Université de Bath, à l'origine du Journal of Industrial Archaeology édité à partir de 1964.

2. Pour revisiter l'histoire de ces échanges, voir Balut 1984 ; Daumas 1982 ; Chassagne 2002.

3. Sur le thème de la reconversion, voir L'archéologie industrielle en France 2007 et Smith 2015.

4. Pour l'histoire du CILAC (Comité d'information et de liaison pour l'archéologie, l'étude et la mise en valeur du patrimoine industriel), voir L'archéologie industrielle en France 2004.

5. Voir, à titre d'exemple, Belot, Lamard 2011 ; Pierrot 2010 ; Woronoff 2003 ; Woronoff 2015.

6. Voir, à titre d'exemple, André 1996; Cardinal et al. 2016; Cotte 2016; Garçon 1998; Garçon, Hilaire-Pérez 2004 ; Hilaire-Pérez 2000 ; Olivier 2004. Voir également la revue Documents pour l'histoire des techniques, CDHTE-CNAM. 
7. Parmi lesquelles, Belot, Lamard 2007 ; Belot, Lamard 2009 ; Daumas 2004 ; Daumas 2006 ; Lohr, Michel et Pierrot 2009.

8. Pour ne citer que quelques exemples : Di Meo 1994 ; Edelbutte 2008 et 2010 ; Garat, GravariBarbas et Veschambre 2001; Gay 2012; Gravari-Barbas et Guichard-Anguis 2003 ; Veschambre 2007 et 2008.

9. A cet égard, le GIEC indique que la mobilisation en faveur de la lutte contre le réchauffement climatique doit aussi s'exercer à l'échelle régionale, voire locale. L'instauration de liens entre les politiques climatiques nationales et infranationales est signalée comme un moyen à ne pas négliger pour optimiser l'atténuation du changement climatique. En ce sens, le rôle clé des institutions politiques locales et du secteur privé est de plus en plus reconnu pour favoriser l'adaptation des communautés et de la société civile à ce nouveau paradigme, (GIEC 2014).

10. Inscription sur la Liste du Patrimoine mondial de l'Humanité au titre de paysage culturel évolutif, le 2 juillet 2012, UNESCO, 36 e session du comité du patrimoine mondial (du 24 juin au 6 juillet 2012, à Saint-Pétersbourg).

11. Spécialiste de l'environnement urbain, professeur émérite d'histoire des techniques au Conservatoire national des arts et métiers (CNAM).

12. Parmi lesquels Jean-Baptiste Fressoz, Frédéric Graber, Thomas Leroux, Michel Letté, Fabien Locher, François Jarrige ou encore Arnaud Péters.

13. Voir les travaux d'Hélène Melin, socio-anthropologue au laboratoire Clersé (UMR 8019), Université Lille 1.

14. Se reporter aux expérimentations menées sur les anciens sites industriels de la Condition publique, à Roubaix ; de l'Union, à Lille ; et de Giat Industrie, actuellement Novacieries, à SaintChamond.

15. Pour plus d'informations sur la rétroconception (reverse-engineering), voir: Cotte 2009, premier travail de synthèse du domaine; Cotte, Laroche 2011; Cotte 2012; Laroche 2007; Laroche et al. 2007 : Michel 2009 ; Hervy 2014.

16. Aujourd'hui maître de conférences HDR en génie mécanique au Laboratoire LS2N, École centrale de Nantes.

17. A titre d'exemple, voir la modélisation 3D du marteau pilon du Creusot effectuée par des étudiants et reprise par une Société de production pour la muséographie du Pavillon de l'Industrie : http://www.afbourdon.com/centre-dinterpretation/

18. https://reseed.ls2n.fr/fr/accueil/.

19. Sylvain Laubé, Ronan Querrec, Marie-Morgan Abiven et Serge Garlatti, Lab In Virtuo : Un environnement Virtuel Intelligent (EVI) participatif dédié à l'histoire et aux patrimoines des paysages culturels industriels. Pour plus d'informations, voir : https://hal.archives-ouvertes.fr/hal-01950624 20. Pour plus d'informations sur Techn'hom Time Machine, voir: https://mshe.univ-fcomte.fr/ poles-de-recherche/dynamiques-territoriales/axe-2-observation-des-territoires-daujourdhui/ 924-ttm-techn-hom-time-machine

21. A cet égard, Wang Shu s'est notamment illustré avec un premier prix décerné en 2007 « Pour une architecture durable ». 


\section{RÉSUMÉS}

Cette leçon sur l'archéologie industrielle vise à porter un regard historiographique sur l'évolution du champ depuis son émergence en France il y a une cinquantaine d'années. Après en avoir défini les contours et retracé la méthodologie, l'objectif est d'apporter de nouvelles clés d'analyse des témoignages matériels et immatériels issus du monde de l'industrie autour des enjeux contemporains. Transition écologique et transition numérique sont au cœur des débats actuels. Comment articuler ces dernières à l'archéologie industrielle ? Cela fait-il d'ailleurs sens et, le cas échéant, quels sont les intérêts en jeu? Dans ces processus de patrimonialisation, la responsabilité tant scientifique que morale de l'historien est engagée. Cette leçon revendique cette double implication. Car si l'archéologie industrielle mobilise les artéfacts du passé, ces derniers peuvent être particulièrement riches et stimulants pour répondre aux préoccupations sociétales actuelles dès lors que la démarche veille à les aborder de manière holistique, dans leur cycle de vie.

This lesson on industrial archaeology seeks to take a historiographical look the evolution of the field since its emergence in France about fifty years ago. After having defined the outlines and traced the methodology, the aim is to provide new keys for the analysis of material and immaterial remains from the world of industry in relation to contemporary challenges. Ecological transition and digital transition are at the centre of current debates. How can they be linked to industrial archaeology? Does this make sense and, if so, what are the interests involved ? In these processes of patrimonialisation, the historian's scientific as well as moral responsibility is engaged. This lesson claims this double implication. If industrial archaeology mobilises the artefacts of the past, they can be particularly rich and stimulating in terms of responding to current societal concerns if the approach ensures that they are approached holistically, in their life cycle.

\section{INDEX}

Mots-clés : histoire des techniques, patrimoine industriel, transition, reconversion, humanités numériques, numérique, archéologie industrielle, soutenabilité

Thèmes : Entretiens et leçons

Keywords : history of technology, industrial heritage, transition, rehabilitation, digital humanities, industrial archaeology, sustainability

\section{AUTEUR}

\section{MARINA GASNIER}

Marina Gasnier est professeur en histoire des techniques et épistémologie du patrimoine industriel à l'Université de technologie Belfort-Montbéliard. Directrice du pôle Humanités depuis 2019, elle exerce ses recherches au sein de RECITS, axe transverse pluridisciplinaire en sciences humaines et sociales de l'Institut FEMTO-ST (UMR 6174). Ses travaux portent sur l'étude des processus de reconversion du patrimoine industriel autour des enjeux contemporains, en particulier environnementaux. Ce faisant, elle s'est engagée dans une réflexion interdisciplinaire mêlant les sciences historiques et celles de l'ingénierie afin d'approfondir la connaissance des matériaux anciens dans la perspective du réemploi du bâti existant. Parallèlement, elle mène une 
autre réflexion interdisciplinaire dans le domaine des humanités numériques et plus précisément celui de « l'archéologie industrielle avancée ». 